\title{
Antidepressants, TMS, and the risk of affective switch in bipolar depression
}

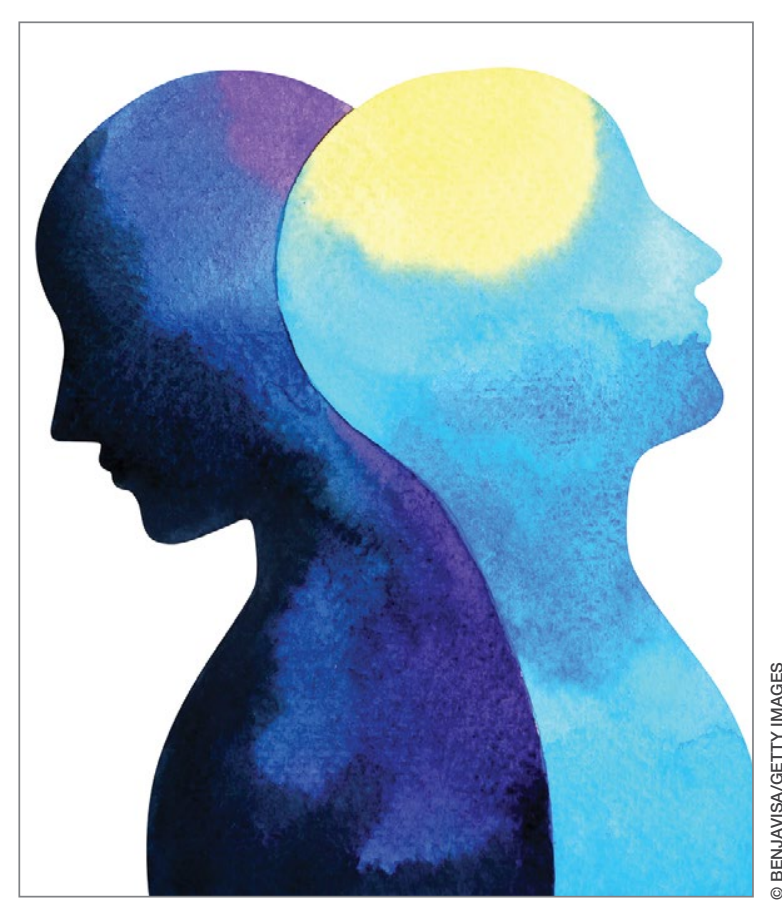

\section{Lucia Smith-Martinez, MD}

Clinical Assistant Professor

Department of Psychiatry and Behavioral Medicine

\section{Kaylan Muppavarapu, MD}

Clinical Assistant Professor

Department of Psychiatry and Behavioral Medicine

\section{Michael Lang, MD}

Assistant Professor

Director, Medicine/Psychiatry Residency Program

Vice Chair

Department of Psychiatry and Behavioral Medicine

....

East Carolina University

Greenville, North Carolina

\section{Some evidence suggests that TMS plus a mood stabilizer might minimize this risk}

B ecause treatment resistance is a pervasive problem in bipolar depression, the use of neuromodulation treatments such as transcranial magnetic stimulation (TMS) is increasing for patients with this disorder. ${ }^{1-7}$ Patients with bipolar disorder tend to spend the majority of the time with depressive symptoms, which underscores the importance of providing effective treatment for bipolar depression, especially given the chronicity of this disease. ${ }^{2,3,5}$ Only a few medications are FDA-approved for treating bipolar depression (Table, page 21).

In this article, we describe the case of a patient with treatment-resistant bipolar depression undergoing adjunctive TMS treatment who experienced an affective switch from depression to mania. We also discuss evidence regarding the likelihood of treatment-emergent mania for antidepressants vs TMS in bipolar depression.

\section{CASE}

Ms. W, a 60-year-old White female with a history of bipolar I disorder and attention-deficit/hyperactivity disorder (ADHD), presented for TMS evaluation during a depressive episode. Throughout her life, she had experienced numerous manic episodes, but as she got older she noted an increasing frequency of depressive episodes. Over the course of her illness, she had completed adequate trials at therapeutic doses of many medications, including second-generation antipsychotics (SGAs) (aripiprazole, lurasidone, olanzapine,

\section{Disclosures}

The authors report no financial relationships with any companies whose products are mentioned in this article, or with manufacturers of competing products.

doi: $10.12788 /$ cp.0099 


\section{Medications that are FDA-approved for treating bipolar depression}

Medication

Brand name

Olanzapine-fluoxetine

Quetiapine

Quetiapine XR

Lurasidone

Cariprazine

XR: extended-release

\begin{tabular}{|c|c|}
\hline Symbyax & 2003 \\
\hline Seroquel & 2006 \\
\hline Seroquel XR & 2008 \\
\hline Latuda & 2013 \\
\hline Vraylar & 2019 \\
\hline
\end{tabular}

quetiapine), mood stabilizers (lamotrigine, lithium), and antidepressants (bupropion, venlafaxine, fluoxetine, mirtazapine, trazodone). A course of electroconvulsive therapy was not effective. Ms. W had a long-standing diagnosis of ADHD and had been treated with stimulants for $>10$ years, although it was unclear whether formal neuropsychological testing had been conducted to confirm this diagnosis. She had $>10$ suicide attempts and multiple psychiatric hospitalizations.

At her initial evaluation for TMS, Ms. W said she had depressive symptoms predominating for the past 2 years, including low mood, hopelessness, poor sleep, poor appetite, anhedonia, and suicidal ideation without a plan. At the time, she was taking clonazepam, $0.5 \mathrm{mg}$ twice a day; lurasidone, $40 \mathrm{mg} / \mathrm{d}$ at bedtime; fluoxetine, $60 \mathrm{mg} / \mathrm{d}$; trazodone, $50 \mathrm{mg} / \mathrm{d}$ at bedtime; and methylphenidate, $40 \mathrm{mg} / \mathrm{d}$, and was participating in psychotherapy consistently.

After Ms. W and her clinicians discussed alternatives, risks, benefits, and adverse effects, she consented to adjunctive TMS treatment and provided written informed consent. The treatment plan was outlined as 6 weeks of daily TMS therapy (NeuroStar; Neuronetics, Malvern, PA), 1 treatment per day, 5 days a week. Her clinical status was assessed weekly using the Quick Inventory of Depressive Symptomatology (QIDS) for depression, Generalized Anxiety Disorder 7-item scale (GAD-7) for anxiety, and Young Mania Rating Scale (YMRS) for mania. The Figure (page 22) shows the trends in Ms. W's QIDS, GAD-7, and YMRS scores over the course of TMS treatment.

Prior to initiating TMS, her baseline scores were QIDS: 25, GAD-7: 9, and YMRS:
7, indicating very severe depression, mild anxiety, and the absence of mania. Ms. W's psychotropic regimen remained unchanged throughout the course of her TMS treatment. After her motor threshold was determined, her TMS treatment began at $80 \%$ of motor threshold and was titrated up to $95 \%$ at the first treatment. By the second treatment, it was titrated up to $110 \%$. By the third treatment, it was titrated up to $120 \%$ of motor threshold, which is the percentage used for the remaining treatments.

Initially, Ms. W reported some improvement in her depression, but this improvement was short-lived, and she continued to have elevated QIDS scores throughout treatment. By treatment \#21, her QIDS and GAD-7 scores remained elevated, and her YMRS score had increased to 12. Due to this increase in YMRS score, the YMRS was repeated on the next 2 treatment days (\#22 and \#23), and her score was 6 on both days. When Ms. W presented for treatment \#25, she was disorganized, irritable, and endorsed racing thoughts and decreased sleep. She was involuntarily hospitalized for mania, and TMS was discontinued. Unfortunately, she did not complete any clinical scales on that day. Upon admission to the hospital, Ms. W reported that at approximately the time of treatment \#21, she had a fluctuation in her mood that consisted of increased goal-directed activity, decreased need for sleep, racing thoughts, and increased frivolous spending. She was treated with lithium, $300 \mathrm{mg}$ twice a day. Lurasidone was increased to $80 \mathrm{mg} / \mathrm{d}$ at bedtime, and she continued clonazepam, trazodone, and methylphenidate at the previous doses. Over 14 days, Ms. W's mania gradually resolved, and she was discharged home.

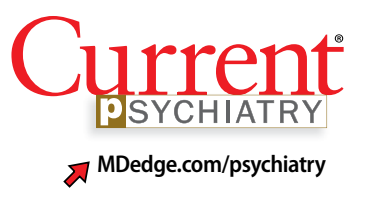

\section{Clinical Point}

The natural course of switching in bipolar disorder confounds interpretation of the evidence about treatment-emergent mania

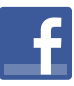

Discuss this article at www.facebook.com/ MDedgePsychiatry 


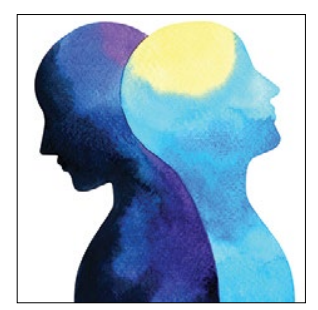

TMS for bipolar depression

\section{Clinical Point}

One review found that the rate of affective switch in patients with bipolar depression who were treated with TMS

\section{was $3.1 \%$}

\section{Figure}

\section{Ms. W's QIDS, GAD-7, and YMRS scores over the course of transcranial magnetic stimulation treatment}

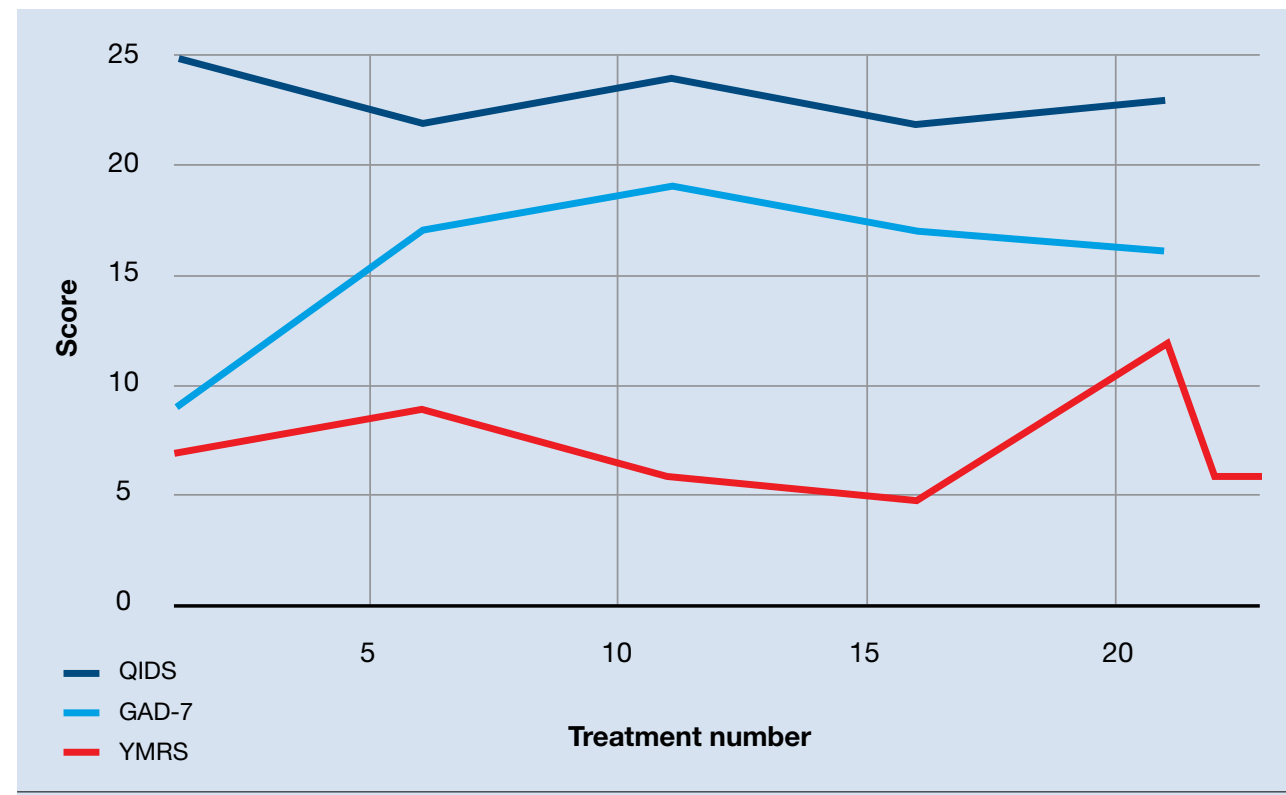

GAD-7: Generalized Anxiety Disorder 7-item scale; QIDS: Quick Inventory of Depressive Symptomatology; YMRS: Young Mania Rating Scale

\section{Mixed evidence on the risk of switching}

Currently, several TMS devices are FDA-cleared for treating unipolar major depressive disorder, obsessive-compulsive disorder, and certain types of migraine. In March 2020, the FDA granted Breakthrough Device Designation for one TMS device, the NeuroStar Advanced Therapy System, for the treatment of bipolar depression. ${ }^{8}$ This designation created an expedited pathway for prioritized FDA review of the NeuroStar Advanced Therapy clinical trial program.

Few published clinical studies have evaluated using TMS to treat patients with bipolar depression. ${ }^{9-15}$ As with any antidepressant treatment for bipolar depression, there is a risk of affective switch from depression to mania when using TMS. Most of the literature available regarding the treatment of bipolar depression focuses on the risk of antidepressant medications to induce an affective switch. This risk depends on the class of the antidepressant, ${ }^{16}$ and there is a paucity of studies examining the risk of switch with TMS.
Interpretation of available literature is limited due to inconsistencies in the definition of an affective switch, the variable length of treatment with antidepressants, the use of concurrent medications such as mood stabilizers, and confounders such as the natural course of switching in bipolar disorder. ${ }^{17}$ Overall, the evidence for treatment-emergent mania related to antidepressant use is mixed, and the reported rate of treatment-emergent mania varies. In a systematic review and meta-analysis of $>20$ randomized controlled trials that included 1,316 patients with bipolar disorder who received antidepressants, Fornaro et $\mathrm{al}^{18}$ found that the incidence of treatment-emergent mania was $11.8 \%$. It is generally recommended that if antidepressants are used to treat patients with bipolar disorder, they should be given with a traditional mood stabilizer to prevent affective switches, although whether mood stabilizers can prevent such switches is unproven. ${ }^{19}$

In a literature review by $\mathrm{Xia}$ et $\mathrm{al}^{20}$ the affective switch rate in patients with bipolar depression who were treated with TMS was $3.1 \%$, which was not statistically different 
from the affective switch rate with sham treatment. However, most of the patients included in this analysis were receiving other medications concurrently, and the length of treatment was 2 weeks, which is shorter than the average length of TMS treatment in clinical practice. In a recent literature review by Rachid, ${ }^{21}$ TMS was found to possibly induce manic episodes when used as monotherapy or in combination with antidepressants in patients with bipolar depression. To reduce the risk of treatment-emergent mania, current recommendations advise the use of a mood stabilizer for a minimum of 2 weeks before initiating TMS. ${ }^{1}$

In our case, Ms. W was receiving antidepressants (fluoxetine and trazodone), lurasidone (an SGA that is FDA-approved for bipolar depression), and methylphenidate before starting TMS treatment. Fluoxetine, trazodone, and methylphenidate may possibly contribute to an increased risk of an affective switch. ${ }^{1,22}$ Further studies are needed to clarify whether mood stabilizers or SGAs can prevent the development of mania in patients with bipolar depression who undergo TMS treatment. ${ }^{20}$

Because bipolar depression poses a major clinical challenge, ${ }^{23,24}$ it is imperative to consider alternate treatments. When evaluating alternative treatment strategies, one may consider TMS in conjunction with a traditional mood stabilizer because this regimen may have a lower risk of treatment-emergent mania compared with antidepressants. ${ }^{1,25}$

\section{Acknowledgment}

The authors thank Dr. Sy Saeed for his expertise and guidance on this article.

\section{References}

1. Aaronson ST, Croarkin PE. Transcranial magnetic stimulation for the treatment of other mood disorders. In: Bermudes RA, Lanocha K, Janicak PG, eds. Transcranial

\section{Related Resources}

- Transcranial magnetic stimulation: clinical applications for psychiatric practice. Bermudes RA, Lanocha K, Janicak PG, eds. American Psychiatric Association Publishing; 2017.

- Gold AK, Ornelas AC, Cirillo P, et al. Clinical applications of transcranial magnetic stimulation in bipolar disorder. Brain Behav. 2019;9(10):e01419. doi: 10.1002/brb3.1419

Drug Brand Names

Bupropion - Wellbutrin

Cariprazine - Vraylar

Clonazepam $\cdot$ Klonopin

Fluoxetine • Prozac

Lamotrigine - Lamictal

Lithium - Eskalith,

Lithobid

Lurasidone - Latuda
Aripiprazole • Abilify

Methylphenidate $\cdot$ Ritalin,
Concerta
Mirtazapine $\cdot$ Remeron
Olanzapine $\cdot$ Zyprexa
Olanzapine-fluoxetine $\cdot$
Symbyax
Quetiapine $\cdot$ Seroquel
Trazodone $\cdot$ Desyrel
Venlafaxine $\cdot$ Effexor

magnetic stimulation: clinical applications for psychiatric practice. American Psychiatric Association Publishing; 2017:127-156.

2. Geddes JR, Miklowitz DJ. Treatment of bipolar disorder. Lancet. 2013;381(9878):1672-1682.

3. Gitlin M. Treatment-resistant bipolar disorder. Molecular Psychiatry. 2006;11(3):227-240.

4. Harrison PJ, Geddes JR, Tunbridge EM. The emerging neurobiology ofbipolardisorder. Trends Neurosci. 2018;41(1): 18-30.

5. Merikangas KR, Jin R, He JP, et al. Prevalence and correlates of bipolar spectrum disorder in the World Mental Health Survey Initiative. Arch Gen Psychiatry. 2011;68(3):241-251

6. Myczkowski ML, Fernandes A, Moreno M, et al. Cognitive outcomes of TMS treatment in bipolar depression: safety data from a randomized controlled trial. J Affect Disord. 2018;235: 20-26.

7. Tavares DF, Myczkowski ML, Alberto RL, et al Treatment of bipolar depression with deep TMS: results from a double-blind, randomized, parallel group, shamcontrolled clinical trial. Neuropsychopharmacology. 2017;42(13):2593-2601.

8. Neuronetics. FDA grants NeuroStar ${ }^{\circledR}$ Advanced Therapy System Breakthrough Device Designation to treat bipolar depression. Accessed February 2, 2021. https://www.globenewswire.com/news-release/2020/ 03/06/1996447/0/en/FDA-Grants-NeuroStar-AdvancedTherapy-System-Breakthrough-Device-Designation-toTreat-Bipolar-Depression.html

9. Cohen RB, Brunoni AR, Boggio PS, et al. Clinical predictors associated with duration of repetitive transcranial magnetic stimulation treatment for remission in bipolar depression: a naturalistic study. J Nerv Ment Dis. 2010;198(9):679-681.

10. Connolly KR, Helmer A, Cristancho MA, et al. Effectiveness of transcranial magnetic stimulation in clinical practice postFDA approval in the United States: results observed with the first 100 consecutive cases of depression at an academic medical center. J Clin Psychiatry. 2012;73(4):e567-e573.

continued

\section{Bottom Line}

For patients with bipolar depression, treatment with transcranial magnetic stimulation in conjunction with a mood stabilizer may have lower rates of treatment-emergent mania than treatment with antidepressants. 


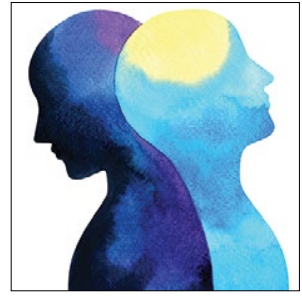

TMS for bipolar depression

\section{Clinical Point}

\section{Compared with} antidepressants, TMS

plus a mood stabilizer may have a lower risk of treatmentemergent mania
11. Dell'osso B, D'Urso N, Castellano F, et al. Long-term efficacy after acute augmentative repetitive transcranial magnetic stimulation in bipolar depression: a 1-year follow-up study. J ECT. 2011;27(2):141-144.

12. Dell'Osso B, Mundo E, D'Urso N, et al. Augmentative repetitive navigated transcranial magnetic stimulation (rTMS) in drug-resistant bipolar depression. Bipolar Disord. 2009;11(1):76-81.

13. Harel EV, Zangen A, Roth $\mathrm{Y}$, et al. H-coil repetitive transcranial magnetic stimulation for the treatment of bipolar depression: an add-on, safety and feasibility study. World J Biol Psychiatry. 2011;12(2):119-126.

14. Nahas Z, Kozel FA, Li X, et al. Left prefrontal transcranial magnetic stimulation (TMS) treatment of depression in bipolar affective disorder: a pilot study of acute safety and efficacy. Bipolar Disord. 2003;5(1):40-47.

15. Tamas RL, Menkes D, El-Mallakh RS. Stimulating research: a prospective, randomized, double-blind, shamcontrolled study of slow transcranial magnetic stimulation in depressed bipolar patients. J Neuropsychiatry Clin Neurosci. 2007;19(2):198-199.

16. Tundo A, Cavalieri P, Navari S, et al. Treating bipolar depression - antidepressants and alternatives: a critical review of the literature. Acta Neuropsychiatrica. 2011: 23(3):94-105.

17. Gijsman HJ, Geddes JR, Rendell JM, et al. Antidepressants for bipolar depression: a systematic review of randomized, controlled trials. Am J Psychiatry. 2004;161(9):1537-1547.
18. Fornaro M, Anastasia A, Novello S, et al. Incidence, prevalence and clinical correlates of antidepressantemergent mania in bipolar depression: a systematic review and meta-analysis. Bipolar Disord. 2018;20(3):195-227.

19. Pacchiarotti I, Bond DJ, Baldessarini RJ, et al. The International Society for Bipolar Disorders (ISBD) task force report on antidepressant use in bipolar disorders. Am J Psychiatry. 2013;170(11):1249-1262.

20. Xia G, Gajwani P, Muzina DJ, et al. Treatment-emergent mania in unipolar and bipolar depression: focus on repetitive transcranial magnetic stimulation. Int J Neuropsychopharmacol. 2008;11(1):119-130.

21. Rachid F. Repetitive transcranial magnetic stimulation and treatment-emergent mania and hypomania: a review of the literature. J Psychiatr Pract. 2017;23(2):150-159.

22. Victorin A, Rydén E, Thase M, et al. The risk of treatmentemergent mania with methylphenidate in bipolar disorder. Am J Psychiatry. 2017;174(4):341-348.

23. Hidalgo-Mazzei D, Berk M, Cipriani A, et al. Treatmentresistant and multi-therapy-resistant criteria for bipolar depression: consensus definition. Br J Psychiatry. 2019;214(1): 27-35.

24. Baldessarini RJ, Vázquez GH, Tondo L. Bipolar depression: a major unsolved challenge. Int J Bipolar Disord. 2020;8(1):1.

25. Phillips AL, Burr RL, Dunner DL. Repetitive transcranial magnetic stimulation in the treatment of bipolar depression: Experience from a clinical setting. J Psychiatr Pract. 2020; 26(1):37-45. 